in all patients. Final staging is summarised in table 1. 4 patients $(9.1 \%)$ with stage II disease did not require further neuroimaging. One patient $(2.3 \%)$ was found to have brain metastases, which were confirmed on MRI. 9 of the patients with stage III disease on PET-CT went on to have negative MRI brain imaging as per national guidelines.

Conclusion Contrast PET-CT is feasible and can provide diagnostic quality CT images of brain and mediastinum. This removes the need for a separate contrast CT brain in patients with stage II disease and enables contrast CT mediastinal images to be obtained following a low dose non contrast initial CT, eg lung cancer screening. Further work is required to assess the additional benefit/cost effectiveness of MRI brain where contrast CT-PET demonstrates stage III disease.

\section{P167 EVALUATION OF EBUS SERVICE-DELIVERY ACROSS THE UK: A NATIONWIDE SURVEY}

${ }^{1} \mathrm{~S}$ Hassan, ${ }^{1} \mathrm{~K}$ Ur Rehman, ${ }^{2} \mathrm{R}$ Baghai-Ravary. 'Guys and St Thomas NHS Foundation Trust, London, UK; ' ${ }^{2}$ East Kent Hospitals University NHS Foundation Trust, Ashford, UK

\subsection{6/thorax-2021-BTSabstracts. 276}

Introduction and Objectives There has been a significant increase in EBUS services across the UK since the recognition of transbronchial needle aspiration (TBNA) guided by endobronchial ultrasound (EBUS) as a key diagnostic step in the diagnosis and staging of suspected lung cancer. ${ }^{1}$

However, there has been no national study to look at how EBUS services are delivered in the UK. The purpose of this study was to assess any variations in the operational setup and practice of EBUS services across the UK.

Methods We conducted an online survey of EBUS practices in the UK between January and March 2020. A response from the EBUS operators was sought from every U.K. Hospital Trust, looking at potential variables in the delivery of EBUS services. One response per site was accepted.

\begin{tabular}{|c|c|c|c|}
\hline & Number (percer & tage) of responses & \\
\hline Type of EBUS session & $\begin{array}{l}\text { Dedicated } \\
31(26.3)\end{array}$ & $\begin{array}{l}\text { Combined with } \\
\text { bronchoscopy } \\
79 \text { (66.9) }\end{array}$ & $\begin{array}{l}\text { Other } \\
8(6.8)\end{array}$ \\
\hline Number of operators & $\begin{array}{l}\text { Single } \\
27(22.8)\end{array}$ & $\begin{array}{l}\text { Two } \\
83(70.3)\end{array}$ & $\begin{array}{l}\text { Variable } \\
8(6.8)\end{array}$ \\
\hline $\begin{array}{l}\text { Second operator, when } \\
\text { present }\end{array}$ & $\begin{array}{l}\text { Consultant } \\
40(44)\end{array}$ & $\begin{array}{l}\text { Trainee or Fellow } \\
48(52.7)\end{array}$ & $\begin{array}{l}\text { Practitioner } \\
3(3.3)\end{array}$ \\
\hline $\begin{array}{l}\text { Number of weekly EBUS } \\
\text { sessions }\end{array}$ & $\begin{array}{l}\text { Less than two } \\
46 \text { (39) }\end{array}$ & $\begin{array}{l}\text { Two to Four } \\
61(51.7)\end{array}$ & $\begin{array}{l}\text { Other } \\
11(9.3)\end{array}$ \\
\hline $\begin{array}{l}\text { Number of patient slots per } \\
\text { list }\end{array}$ & $\begin{array}{l}\text { Less than two } \\
8(6.8)\end{array}$ & $\begin{array}{l}\text { Two to Four } \\
100(84.7)\end{array}$ & $\begin{array}{l}\text { More than four } \\
10(8.5)\end{array}$ \\
\hline Allocated time per case & $\begin{array}{l}<45 \text { minutes } \\
5(4.2)\end{array}$ & $\begin{array}{l}45-60 \text { minutes } \\
110(93.2)\end{array}$ & $\begin{array}{l}>60 \text { minutes } \\
3(2.5)\end{array}$ \\
\hline Cyto-pathologist present & $\begin{array}{l}\text { Yes } \\
12(10.2)\end{array}$ & $\begin{array}{l}\text { No } \\
106(89.8)\end{array}$ & \\
\hline Suspension medium & $\begin{array}{l}\text { Normal saline } \\
26(25.0)\end{array}$ & $\begin{array}{l}\text { Formalin } \\
37(35.6)\end{array}$ & $\begin{array}{l}\text { Formalin and saline } \\
32(30.8) \\
\text { Others } 9(8.7)\end{array}$ \\
\hline Slides prepared & Yes 18 (15.3) & No $100(84.7)$ & \\
\hline
\end{tabular}

Results 218 responses by senior clinicians were received from 118 centres delivering EBUS service in England, Wales, Scotland, and Northern Ireland. Results are summarised in table 1.

Discussion More than two thirds of the centres deliver EBUS in sessions combined with bronchoscopy, whilst $61 \%$ of centres offered at least two weekly sessions; There may be cost-effectiveness in pooling EBUS into dedicated sessions.

In more than $70 \%$ of cases, two operators were utilised whilst a single operator performed EBUS in only $23 \%$ of centres; More than 50\% of the hospitals used a trainee or fellow to assist in the procedure, demonstrating a range of operational practices.

Almost $90 \%$ of trusts had no pathologists to guide realtime sampling. The predominant medium used for suspension of cytopathological samples was formalin (35.6\%), reducing degradation of cytopathological samples compared to normal saline.

Conclusion This survey demonstrates notable differences in EBUS practice across the UK, which could result in variation in cost-effectiveness, quality, and safety; A national consultation group may allow dissemination of best-practice.

\section{REFERENCE}

1. National Institute for Health and Care Excellence. Endobronchial ultrasound guided transbronchial needle aspiration for mediastinal masses. Interventional procedures guidance, 27 February 2008.

\section{P168 ENDOBRONCHIAL ULTRASOUND-GUIDED TRANSBRONCHIAL NEEDLE ASPIRATION FOR ASYMPTOMATIC OR INCIDENTAL BILATERAL HILAR OR MEDIASTINAL ADENOPATHY: AN UNNECESSARY TEST?}

A Begbey, J Karimjee, N Piletska, A Nasir, T Loke. Maidstone and Tunbridge Wells NHS Trust, Tunbridge Wells, UK

\subsection{6/thorax-2021-BTSabstracts. 277}

Introduction and Objectives There is a lack of consensus regarding the need for biopsy in patients presenting with asymptomatic bilateral hilar or mediastinal adenopathy (BHMA). The American Thoracic Society suggests close follow up a 'reasonable alternative' to biopsy. The British Thoracic Society suggests multidisciplinary input in atypical clinical or radiological cases.

Our hypothesis was that in our population endobronchial ultrasound-guided transbronchial needle aspiration (EBUSTBNA) provides little diagnostic value over Computerised Tomography (CT) of the Thorax in the investigation of asymptomatic or incidental BHMA.

Method We conducted a retrospective analysis at our NHS Trust, assessing whether EBUS-TBNA revealed occult malignancy or unexpected pathology in patients presenting with asymptomatic or incidental BHMA on CT.

Inclusion criteria included a radiological diagnosis of likely sarcoid with bilateral hilar lymphadenopathy and/or non-specific adenopathy with no other lung parenchymal abnormality. Exclusion criteria included cases of known malignancy or radiological features of malignancy as reported by a radiologist.

Results Between September 2016 to August 2019, a total of 366 EBUS-TBNA procedures were performed, of which 80 cases met the inclusion criteria. Adequate TBNA samples were obtained in 72/80 (90\%). 57 cases had features consistent 
with sarcoidosis and 13 samples showed benign or reactive features. 1 sample was suspicious for lymphoma on TBNA (this was later excluded by further investigations). 1 patient had EBUS-TBNA showing non-caseating granulomas but was subsequently re-evaluated for thrombocytopaenia and hyperferritinaemia. A lymphoma was later confirmed by extrathoracic lymph node biopsy.

Of the 8 cases where initial EBUS-TBNA samples were inadequate, 5 were deemed benign following multidisciplinary team assessment, subsequent biopsy or surveillance. 3/8 cases underwent a further EBUS-TBNA showing features consistent with sarcoidosis.

Conclusion In our population, EBUS-TBNA in asymptomatic individuals with BHMA rarely results in a diagnosis of malignancy or significant pathology. Careful evaluation combined with CT surveillance may be a suitable alternative to early EBUS/TBNA in this cohort of patients.

\section{REFERENCES}

1. Diagnosis and Detection of Sarcoidosis, Crouser, et al., American Journal of Respiratory and Critical Care Medicine, Volume 201, Issue 8, 4/15/20

2. BTS Clinical Statement on pulmonary sarcoidosis, Thillai, et al. Thorax 2/12/20

\section{P169 AN UPDATE ON THE STRATIFY (STAGING BY THORACOSCOPY IN POTENTIALLY RADICALLY TREATABLE NON-SMALL CELL LUNG CANCER ASSOCIATED WITH MINIMAL PLEURAL EFFUSION) STUDY}

\begin{abstract}
1J Ferguson, ${ }^{2} \mathrm{~N}$ Rahman, ${ }^{3} \mathrm{~N}$ Maskell, ${ }^{4} \mathrm{~J}$ Lyons, ${ }^{5} \mathrm{~S}$ Grundy, ${ }^{6} \mathrm{D}$ Sivakumar, ${ }^{7} \mathrm{~J}$ Corcoran, ${ }^{8} \mathrm{D}$ Menzies, ${ }^{9} \mathrm{~A}$ Chalmers, ${ }^{10} \mathrm{~L}$ Alexander, ${ }^{10} \mathrm{C}$ Kelly, ${ }^{10} \mathrm{~A}$ Shaw, ${ }^{11} \mathrm{C}$ Dick, ${ }^{12} \mathrm{~S}$ Tsim, ${ }^{13} \mathrm{G}$ Cowell, ${ }^{14} \mathrm{~T}$ Hopkins, ${ }^{14} \mathrm{R}$ Woodward, ${ }^{15} \mathrm{KG}$ Blyth. ${ }^{1} \mathrm{G}$ lasgow Pleural Disease Unit, Queen Elizabeth University Hospital, Glasgow, UK; ${ }^{2}$ Oxford Respiratory Trials Unit, Oxford Raddliffe Hospital, Oxford, UK; ${ }^{3}$ Academic Respiratory Unit, Southmead Hospital, Bristol, UK; ${ }^{4}$ Wythenshawe Hospital, Manchester University, Manchester, UK; ${ }^{5}$ Salford Royal Hospital, Salford, UK; ${ }^{6}$ GSTT NHS Foundation Trust, London, UK; ' University Hospital Plymouth, Plymouth, UK; ${ }^{8}$ Glan Clwyd Hospital, Rhyl, UK; ${ }^{9}$ Beatson Institute for Cancer Research, Glasgow, UK; ${ }^{10}$ Cancer Research UK Clinical Trials Unit, Glasgow, UK; ${ }^{11}$ Department of Pathology, Queen Elizabeth University Hospital, Glasgow, UK; ${ }^{12}$ Department of Respiratory Medicine, Queen Elizabeth University Hospital, Glasgow, UK; ${ }^{13}$ Department of Radiology, Queen Elizabeth University Hospital, Glasgow, UK; ${ }^{14}$ Glasgow Clinical Research Imaging Facility, Queen Elizabeth University Hospital, Glasgow, UK; ${ }^{15}$ Institute of Cancer Sciences, Glasgow, UK
\end{abstract}

\subsection{6/thorax-2021-BTSabstracts.278}

Introduction Pleural effusion is common in lung cancer. Metastatic disease may be confirmed on imaging or fluid sampling. A minority of patients however with otherwise radically treatable disease have a small effusion not amenable to aspiration, or from which fluid cytology is negative; termed minimal pleural effusion (mini-PE). Previous retrospective studies associate significantly shorter survival in mini-PE than stage-matched cases without mini-PE and hypothesise this reflects occult pleural metastases (OPM) in up to $80 \%$ of patients. STRATIFY (Staging by Thoracoscopy in Potentially Radically Treatable Non-Small Cell Lung Cancer (NSCLC) Associated with Minimal Pleural Effusion) is a multicentre, prospective observational study, which will determine the true prevalence of OPM in this setting. An update on the study is provided here.

Methods STRATIFY was funded by Chief Scientist Office and opened to recruitment in Jan-20. Target $n=96$ across 8 UK centres in 18 months. Key eligibility criteria include Mini-PE (defined by an ipsilateral effusion $<1 / 3$ hemithorax on chest radiograph), radically treatable NSCLC and LAT feasibility (defined by sufficient fluid \pm lung sliding on screening ultrasound). Primary endpoint: Prevalence of OPM, defined as NSCLC cells in parietal pleural biopsies. Key secondary endpoints include LAT safety, the impact of LAT results on NSCLC treatment plans and non-invasive MRI-derived measures of cardiac function and altered body composition (as alternative explanations for mini-PE). Study progress, including the impact of COVID19 was reviewed and summarised.

Results STRATIFY was rapidly halted due to COVID19 after 1 patient was recruited. The study was allowed to reopen in July-20 but given a dramatic reduction in lung cancer referrals across the UK and delayed site set up processes, the study team took the decision to close recruitment from Oct-20 to Apr-21. This was supported by the funder who provided a costed 6-month extension. By June-21, 4/8 sites have opened. $4 / 6$ six screened patients have been recruited, $2 / 4$ have entered the MRI sub-study.

Conclusions STRATIFY will determine the true prevalence of OPM in patients with radically treatable NSCLC and mini-PE. The study outcomes will be important in defining an extended role for LAT as a pleural staging tool.

\section{P170 PLEURAL RECURRENCE AFTER TRANSTHORACIC NEEDLE LUNG BIOPSY IN STAGE I LUNG CANCER}

K Jackson, O Kent, C Storey, E Pang, M Hadi, S VijayaGopal, A Aujayeb. Northumbria Healthcare NHS Foundation Trust, Newcastle upon Tyne, UK

\subsection{6/thorax-2021-BTSabstracts.279}

Introduction Hong et al determined, from 2394 patients with stage 1 lung cancer, that needle or intra-operative transthoracic biopsy had a higher risk for pleural recurrence. ${ }^{1}$ There was local concern that a straight to surgery approach advocated might not be sustainable and/or warranted, that the data might only apply to Asian countries, and that there were missing values for microscopic invasion.

Methods An analysis of all Stage 1 lung cancers enrolled onto the Somerset cancer register was performed (Caldicott ref 3756). Exclusion criteria were mesothelioma, non-lung cancers and non-diagnostic biopsies. Data collected were demographics, diagnostic procedures, operation, pathology, CT findings, time to recurrence, recurrence type, survival and time to death.

Results 493 patients with stage 1 cancer were identified (Jan 2013-Dec 2020). Data was insufficient in 34.169 patients had a positive CT guided or pre-operative biopsy: mean age 73 years (range 48-97) and 105 (60\%) females. Diagnoses were predominantly 99 (57\%) adenocarcinomas and 49 (28\%) squamous cell cancers. Any recurrence occurred in 42 ( $24 \%$ vs $19 \%$ with Hong et $\mathrm{al}^{1}$ ) patients and concomitant ipsilateral pleural recurrence in 10 (6\%-similar). Of those 10 , 8 underwent CT guided biopsies, and 2 pre-operative biopsies, 8 were male, 2 female and 50\% (5) were adenocarcinomas. 8 were solid tumours, and 6 had pleural contract. Lympho-vascular-pleural invasion was present in 6 of those 10 patients. Mean time to recurrence was 8.8 months (4-18) and mean time from recurrence to death 8.1 months (1-26). 210 patients had no biopsies, mean age was 77 years (4999). Any recurrence occurred in 32 (15\%); pleural recurrence in $2(1 \%)$, mean time 19 months. 2 patients in this 